\title{
Application of Generalized Fractional Thermoelasticity Theory with Two Relaxation Times to an Electromagnetothermoelastic Thick Plate
}

\author{
A. M. Abd El-Latief \\ Department of Mathematics, Alexandria University, Alexandria, Egypt \\ Correspondence should be addressed to A. M. Abd El-Latief; m.abdellatief@yahoo.com
}

Received 19 February 2016; Revised 18 July 2016; Accepted 25 August 2016

Academic Editor: Santiago Garcia-Granda

Copyright (C) 2016 A. M. Abd El-Latief. This is an open access article distributed under the Creative Commons Attribution License, which permits unrestricted use, distribution, and reproduction in any medium, provided the original work is properly cited.

\begin{abstract}
The fractional mathematical model of Maxwell's equations in an electromagnetic field and the fractional generalized thermoelastic theory associated with two relaxation times are applied to a $1 \mathrm{D}$ problem for a thick plate. Laplace transform is used. The solution in Laplace transform domain has been obtained using a direct method and its inversion is calculated numerically using a method based on Fourier series expansion technique. Finally, the effects of the two fractional parameters (thermo and magneto) on variable fields distributions are made. Numerical results are represented graphically.
\end{abstract}

\section{Introduction}

Fractional calculus (FC) is a very useful tool in describing the evolution of systems with memory, which typically are dissipative and complex systems such as glasses, biopolymers, biological cells, porous materials, amorphous semiconductors, and liquid crystals. Scaling laws and self-similar behavior are supposed to be fundamental features of complex systems. In recent decades the FC and in particular the fractional differential equations have attracted interest of researchers in several areas including mathematics, physics, chemistry, biology, engineering, and economics [1-4].

FC theory has been used successfully in thermoelasticity and thermoviscoelasticity, such that a quasi-static uncoupled theory of thermoelasticity based on the fractional heatconduction equation was put forward by Povstenko [5]. The theory of thermal stresses based on the heat-conduction equation with the Caputo time-fractional derivative is used by Povstenko [6] to investigate thermal stresses in an infinite body with a circular cylindrical hole. Sherief et al. [7] introduced a fractional order theory of thermoelasticity. Raslan $[8,9]$ has solved $1 \mathrm{D}$ problems in the context of this theory and applied this theory to $2 \mathrm{D}$ problem of thick plate [10]. The fractional parameter effect of this theory on thermoelastic material with variable thermal variable thermal material properties has been studied in [11, 12]. Ezzat [13] established a model of fractional heat-conduction equation by using the Taylor series expansion of time-fractional order developed by Jumarie [14].

Hamza et al. established a new mathematical model of Maxwell's equations in an electromagnetic field in [15] and derived a fractional model for thermoelasticity associated with two relaxation times in [16]. A model for unsteady thermoelectric magnetohydrodynamics (TEMHD) flow and heat transfer of two immiscible second-grade fluids with two fractional parameters was introduced in [17].

The previous work introduced by Hamza et al. [18] describes one-dimensional problems in the context of the theory [16] in which the electromagnetic field effects are ignored. The model in the previous work depends only upon one fractional parameter $\alpha$ which does not have electric or magnetic affects. Another application of this theory introduced in [16] depends on two fractional parameters $\alpha$ and $\beta$. The first fractional parameter $\alpha$ appears only in the heat equation and is absent from both the equation of motion and the constitutive equations. In this work, we solve a $1 \mathrm{D}$ problem for a thick plate that was not solved in the context of both theories $[15,16]$.

The new model here depends on two fractional parameters $\alpha$ and $\beta$. The first fractional parameter $\alpha$ appears in the 
heat equation, the equation of motion, and the constitutive equations.

The effects of the fractional parameters corresponding to two models $[15,16]$ are discussed. The solution is obtained in Laplace transformed domain using a direct approach. All the studied field variables are represented graphically.

\section{The Mathematical Model}

The governing fractional Maxwell's equations in an electromagnetic field are given by [15]

$$
\begin{aligned}
\nabla \times \mathbf{H} & =\mathbf{J}+\varepsilon_{0} \frac{\partial \mathbf{E}}{\partial t}, \\
\nabla \times \mathbf{E} & =-\mu_{0} \frac{\tau^{\beta-1}}{\beta !} \frac{\partial^{\beta} \mathbf{H}}{\partial t^{\beta}}, \\
\nabla \cdot \mathbf{H} & =0 \\
\nabla \cdot \mathbf{E} & =0,
\end{aligned}
$$

where $\beta$ is the order of the fractional derivative in Caputo sense with respect to time $t$ such that $0 \leq \beta \leq 1, \mu_{0}$ is the magnetic permeability, and $\varepsilon_{0}$ is the electric permeability. $\mathbf{H}$ and $\mathbf{E}$ are the magnetic and electric field intensities, respectively. $\mathbf{J}$ is the current density and $\tau$ is a positive constant.

Equation (2) is called "Fractional Faraday's Law of Magnetic Induction" which was derived in [15]. The proof uses Faraday's induction law and the fractional Taylor's series expansion developed by Jumarie [14].

Ohm's law for moving media states that

$$
\mathbf{J}=\sigma_{0}\left[\mathbf{E}+\mu_{0} \frac{\partial \mathbf{u}}{\partial t} \times \mathbf{H}\right]
$$

where $\sigma_{0}$ is the electric conductivity of the medium (assumed to be infinite) and $\mathbf{u}$ is the displacement vector.

Since $\mathbf{J}$ is bounded and $\sigma_{0}$ is infinite, it follows that

$$
\mathbf{E}=-\mu_{0} \frac{\partial \mathbf{u}}{\partial t} \times \mathbf{H}
$$

The governing equations for generalized fractional thermoelasticity associated with two relaxation times in the absence of external body forces and heat sources are given by [16] the following.

(i) The constitutive equations:

$$
\sigma_{i j}=2 \mu e_{i j}+\lambda \delta_{i j} e_{k k}-\beta_{1} \delta_{i j}\left(1+\frac{\tau_{1}^{\alpha}}{\alpha !} \frac{\partial^{\alpha}}{\partial t^{\alpha}}\right) \theta,
$$

where $\theta$ is the temperature of the medium, $\sigma_{i j}$ are the components of the stress tensor, the constants $\lambda$ and $\mu$ are Lamés constants, and $\beta_{1}=\alpha_{t}(3 \lambda+2 \mu)$, where $\alpha_{t}$ is the coefficient of linear thermal expansion and $\alpha \in(0,1]$ is the order of the time-fractional derivative. $\tau_{1}$ is a positive constant and $e_{i j}$ are the components of the strain tensor.

(ii) The strain-displacement relations:

$$
e_{i j}=\frac{1}{2}\left(u_{i, j}+u_{j, i}\right) \text {. }
$$

(iii) The equation of motion:

$$
\sigma_{i j, j}+F_{i}=\rho \ddot{u}_{i}
$$

where $\rho$ is the density assumed independent of time $t, u_{i}$ is the displacement vector component, $\sigma_{i j}$ are the components of the stress tensor, and $F_{i}$ is the component of the Lorentz force given by

$$
F_{i}=\mu_{0}(\mathbf{J} \times \mathbf{H})_{i} .
$$

(iv) The fractional heat equation:

$$
k \theta_{, i i}=\beta_{1} \theta_{0} \dot{e}_{k k}+\rho C_{E}\left(\frac{\partial}{\partial t}+\frac{\tau_{0}^{\alpha}}{\alpha !} \frac{\partial^{1+\alpha}}{\partial t^{1+\alpha}}\right) \theta,
$$

where the specific heat at constant strain is $C_{E}$ and $k$ is the thermal conductivity. $\tau_{0}$ is a positive constant and $\theta_{0}$ is a reference temperature assumed to be such that $\left|\left(\theta-\theta_{0}\right) / \theta_{0}\right| \ll$ 1. As usual, superimposed dots denote time derivatives. The convention of summing over repeated indices is used.

\section{Formulation of the Problem}

We consider a magnetothermoelastic thick plate of perfect conductivity occupying the region $0 \leq x \leq \ell$ in an initial magnetic field $\mathbf{H}_{0}$ in $y$ direction at a uniform reference temperature $\theta_{0}$. This produces an induced magnetic field $\mathbf{h}$ in the direction of the $y$-axis and an electric field $\mathbf{E}$ in the $z$-axis (perpendicular to $\mathbf{H}$ and $\mathbf{u}$ ).

The $x$-axis is perpendicular to the surface of the plate. The upper surface $(x=0)$ of the plate is taken to be traction-free and is subjected to a thermal shock that is a function of time. The lower surface $(x=\ell)$ of the plate is taken to be thermally isolated and laid on a rigid foundation.

It is assumed that all the state functions depend on $x$ and $t$ only.

Thus

$$
\begin{aligned}
\mathbf{u} & =(u(x, t), 0,0), \\
\mathbf{H} & =\left(0, H_{0}+h, 0\right), \\
\mathbf{E} & =(0,0, E) .
\end{aligned}
$$

In the context of generalized fractional thermoelasticity associated with two relaxation times, the constitutive equations, the equation of motion, and fractional heat equation can be expressed in our case as follows:

$$
\begin{aligned}
& \sigma=\sigma_{x x}=(2 \mu+\lambda) \frac{\partial u}{\partial x}-\beta_{1}\left(1+\frac{\tau_{1}^{\alpha}}{\alpha !} \frac{\partial^{\alpha}}{\partial t^{\alpha}}\right) \theta, \\
& e_{x x}=\frac{\partial u}{\partial x}, \\
& \rho \frac{\partial^{2} u}{\partial t^{2}}+F_{1}=(2 \mu+\lambda) \frac{\partial^{2} u}{\partial x^{2}}-\beta_{1}\left(1+\frac{\tau_{1}^{\alpha}}{\alpha !} \frac{\partial^{\alpha}}{\partial t^{\alpha}}\right) \frac{\partial \theta}{\partial x}, \\
& k \frac{\partial^{2} \theta}{\partial x^{2}}=\frac{\partial}{\partial t}\left[\beta_{1} \theta_{0}\left(\frac{\partial u}{\partial x}\right)+\rho C_{E}\left(1+\frac{\tau_{0}^{\alpha}}{\alpha !} \frac{\partial^{\alpha}}{\partial t^{\alpha}}\right) \theta\right] .
\end{aligned}
$$


Now, (1)-(4b) yield

$$
\begin{aligned}
& J=\frac{\partial h}{\partial x}-\varepsilon_{0} \frac{\partial E}{\partial t} \\
& h=-\frac{H_{0} \beta !}{\tau^{\beta-1}} \frac{\partial^{1-\beta}}{\partial t^{1-\beta}}\left(\frac{\partial u}{\partial x}\right), \\
& E=-\mu_{0} H_{0} \frac{\partial u}{\partial t} .
\end{aligned}
$$

From (13), (14), and (12), we obtain

$$
J=\varepsilon_{0} \mu_{0} H_{0}\left(\frac{\partial^{2} u}{\partial t^{2}}\right)-\frac{H_{0} \beta !}{\tau^{\beta-1}} \frac{\partial^{1-\beta}}{\partial t^{1-\beta}}\left(\frac{\partial^{2} u}{\partial x^{2}}\right) .
$$

From (13), (15), and (8), we obtain

$$
F_{1}=\mu_{0} H_{0}^{2}\left(\frac{\beta !}{\tau^{\beta-1}} \frac{\partial^{1-\beta}}{\partial t^{1-\beta}}\left(\frac{\partial^{2} u}{\partial x^{2}}\right)-\varepsilon_{0} \mu_{0}\left(\frac{\partial^{2} u}{\partial t^{2}}\right)\right)
$$

We shall use the following nondimensional variables

$$
\begin{aligned}
\left(x^{\prime}, u^{\prime}\right) & =c \eta(x, u), \\
\left(t^{\prime}, \tau_{0}^{\prime}, \tau_{1}^{\prime}, \tau^{\prime}\right) & =c^{2} \eta\left(t, \tau_{0}, \tau_{1}, \tau\right), \\
\sigma^{\prime} & =\frac{\sigma}{\lambda+2 \mu}, \\
\theta^{\prime} & =\frac{\beta_{1}\left(\theta-\theta_{0}\right)}{\lambda+2 \mu}, \\
h^{\prime} & =\frac{h}{H_{0}}, \\
E^{\prime} & =\frac{E}{\mu_{0} H_{0} c} .
\end{aligned}
$$

Now, using the above nondimensional variables, the system of equations of the problem will reduce to

$$
\begin{aligned}
\left(1+\alpha_{0}^{2}\right) \frac{\partial^{2} u}{\partial t^{2}}= & \left(1+R_{H} \beta ! \tau^{1-\beta} \frac{\partial^{1-\beta}}{\partial t^{1-\beta}}\right)\left(\frac{\partial^{2} u}{\partial x^{2}}\right) \\
& -\left(1+\frac{\tau_{1}^{\alpha}}{\alpha !} \frac{\partial^{\alpha}}{\partial t^{\alpha}}\right) \theta,
\end{aligned}
$$

$$
\begin{aligned}
\frac{\partial^{2} \theta}{\partial x^{2}} & =\frac{\partial}{\partial t}\left[\varepsilon\left(\frac{\partial u}{\partial x}\right)+\left(1+\frac{\tau_{0}^{\alpha}}{\alpha !} \frac{\partial^{\alpha}}{\partial t^{\alpha}}\right) \theta\right], \\
e_{x x} & =\frac{\partial u}{\partial x} \\
h & =-\beta ! \tau^{1-\beta} \frac{\partial^{1-\beta}}{\partial t^{1-\beta}}\left(\frac{\partial u}{\partial x}\right), \\
E & =-\frac{\partial u}{\partial t}, \\
\sigma & =\frac{\partial u}{\partial x}-\left(1+\frac{\tau_{1}^{\alpha}}{\alpha !} \frac{\partial^{\alpha}}{\partial t^{\alpha}}\right) \theta,
\end{aligned}
$$

where

$$
\begin{aligned}
c & =\sqrt{\frac{\lambda+2 \mu}{\rho}}, \\
\eta & =\frac{\rho c_{E}}{k}, \\
R_{H} & =\frac{\mu_{0} H_{0}}{\rho c^{2}}, \\
\alpha_{0} & =\frac{\mu_{0} \varepsilon_{0} H_{0}}{\rho}, \\
\varepsilon & =\frac{T_{0} \beta_{1}^{2}}{(\lambda+2 \mu) k \eta} .
\end{aligned}
$$

Also, we assume that the medium is initially at rest and the undisturbed state is maintained at uniform reference temperature. Then we have

$$
u=u(x, 0)=\dot{u}(x, 0)=\theta(x, 0)=\dot{\theta}(x, 0)=0 .
$$

Now, taking the Laplace transform (denoted by an overbar) with parameter $s$ of both sides of the above system of equations, we get

$$
\begin{aligned}
\left(1+\alpha_{0}^{2}\right) s^{2} \bar{u}= & \left(1+R_{H} \beta ! \tau^{1-\beta} s^{1-\beta}\right)\left(\frac{\partial^{2} \bar{u}}{\partial x^{2}}\right) \\
& -\left(1+\frac{\tau_{1}^{\alpha} s^{\alpha}}{\alpha !}\right) \bar{\theta} \\
\frac{\partial^{2} \theta}{\partial x^{2}}= & s\left[\varepsilon\left(\frac{\partial \bar{u}}{\partial x}\right)+\left(1+\frac{\tau_{0}^{\alpha} s^{\alpha}}{\alpha !}\right) \bar{\theta}\right], \\
\bar{e}_{x x}= & \frac{\partial \bar{u}}{\partial x}, \\
\bar{h}= & -\beta ! \tau^{1-\beta} s^{1-\beta}\left(\frac{\partial \bar{u}}{\partial x}\right), \\
\bar{E}= & -s \bar{u}, \\
\bar{\sigma}= & \frac{\partial \bar{u}}{\partial x}-\left(1+\frac{\tau_{1}^{\alpha} s^{\alpha}}{\alpha !}\right) \bar{\theta} .
\end{aligned}
$$


Solving (21) and (22), we obtain

$$
\left\{\frac{d^{4}}{d x^{4}}+L_{2} \frac{d^{2}}{d x^{2}}+L_{1} \frac{d}{d x}+L_{0}\right\}\{\bar{\theta}, \bar{u}\}=0 .
$$

The corresponding characteristic equation of (27) is

$$
k^{4}+L_{2} k^{2}+L_{1} k+L_{0}=0
$$

where

$$
\begin{aligned}
& L_{2} \\
& =-\frac{s\left(1+\tau_{0}^{\alpha} s^{\alpha} / \alpha !\right)\left(1+R_{H} \beta ! \tau^{1-\beta} s^{1-\beta}\right)+\left(1+\alpha_{0}^{2}\right) s^{2}}{\left(1+R_{H} \beta ! \tau^{1-\beta} s^{1-\beta}\right)}, \\
& L_{1}=-\frac{\varepsilon s\left(1+\tau_{1}^{\alpha} s^{\alpha} / \alpha !\right)}{\left(1+R_{H} \beta ! \tau^{1-\beta} s^{1-\beta}\right)} \\
& L_{0}=-\frac{s^{3}\left(1+\tau_{0}^{\alpha} s^{\alpha} / \alpha !\right)\left(1+\alpha_{0}^{2}\right)}{\left(1+R_{H} \beta ! \tau^{1-\beta} s^{1-\beta}\right)} .
\end{aligned}
$$

The solution of (27) compatible with (22) has the form

$$
\begin{aligned}
& \bar{\theta}=\sum_{i=1}^{4} \varepsilon s k_{i} C_{i} e^{k_{i} x}, \\
& \bar{u}=\sum_{i=1}^{4}\left[k_{i}^{2}-s\left(1+\frac{\tau_{0}^{\alpha} s^{\alpha}}{\alpha !}\right)\right] C_{i} e^{k_{i} x} .
\end{aligned}
$$

Substituting (30) and (31) into (26), we get

$\bar{\sigma}$

$$
=\sum_{i=1}^{4} k_{i}\left[k_{i}^{2}-s\left(1+\frac{\tau_{0}^{\alpha} s^{\alpha}}{\alpha !}\right)-\varepsilon s\left(1+\frac{\tau_{1}^{\alpha} s^{\alpha}}{\alpha !}\right)\right] C_{i} e^{k_{i} x}
$$

Using (31), (23)-(25) become

$$
\begin{aligned}
\bar{e}_{x x} & =\sum_{i=1}^{4} k_{i}\left[k_{i}^{2}-s\left(1+\frac{\tau_{0}^{\alpha} s^{\alpha}}{\alpha !}\right)\right] C_{i} e^{k_{i} x}, \\
\bar{h} & =-\beta !(\tau s)^{1-\beta} \sum_{i=1}^{4} k_{i}\left[k_{i}^{2}-s\left(1+\frac{\tau_{0}^{\alpha} s^{\alpha}}{\alpha !}\right)\right] C_{i} e^{k_{i} x}, \\
\bar{E} & =-s \sum_{i=1}^{4}\left[k_{i}^{2}-s\left(1+\frac{\tau_{0}^{\alpha} s^{\alpha}}{\alpha !}\right)\right] C_{i} e^{k_{i} x} .
\end{aligned}
$$

where $f(t)$ is a known function of $t$. Equations (30)-(32) give

$$
\begin{aligned}
& \sum_{i=1}^{4} \varepsilon s k_{i} C_{i}=\bar{f}(s), \\
& \sum_{i=1}^{4} k_{i}^{2} C_{i} e^{k_{i} \ell}=0, \\
& \sum_{i=1}^{4}\left[k_{i}^{2}-s\left(1+\frac{\tau_{0}^{\alpha} s^{\alpha}}{\alpha !}\right)\right] C_{i} e^{k_{i} \ell}=0, \\
& \sum_{i=1}^{4} k_{i}\left[k_{i}^{2}-s\left(1+\frac{\tau_{0}^{\alpha} s^{\alpha}}{\alpha !}\right)-\varepsilon s\left(1+\frac{\tau_{1}^{\alpha} s^{\alpha}}{\alpha !}\right)\right] C_{i}=0 .
\end{aligned}
$$

\section{Inversion of the Laplace Transform}

We shall now outline the method used to invert the Laplace transforms in the above equations. Let $\bar{f}(s)$ be the Laplace transform of a function $f(t)$. The inversion formula for Laplace transforms can be written as [19]

$$
f(t)=\frac{e^{\gamma t}}{2 \pi} \int_{-\infty}^{\infty} e^{i t y} \bar{f}(\gamma+i y) d y
$$

where $\gamma$ is an arbitrary real number greater than all the real parts of the singularities of $\bar{f}(s)$. Expanding the function $h(t)=\exp (-\gamma t) f(t)$ in a Fourier series in the interval $[0,2 L]$, we obtain the approximate formula [19]:

$$
f(t) \approx f_{N}(t)=\frac{1}{2} c_{0}+\sum_{k=1}^{N} c_{k}, \quad \text { for } 0 \leq t \leq 2 L,
$$

where

$$
c_{k}=\frac{e^{\gamma t}}{L} \operatorname{Re}\left[e^{i k \pi t / L} \bar{f}\left(\gamma+\frac{i k \pi}{L}\right)\right] .
$$

Two methods are used to reduce the total error. First, the "Korrektur" method is used to reduce the discretization error. Next, $\varepsilon$-algorithm is used to reduce the truncation error and therefore to accelerate convergence.

The Korrektur method uses the following formula to evaluate the function $f(t)$ :

$$
f(t)=f_{N K}(t)=f_{N}(t)-e^{-2 \gamma L} f_{N^{\prime}}(2 L+t) .
$$

We shall now describe $\varepsilon$-algorithm that is used to accelerate the convergence of the series in (37). Let $N$ be an odd natural number and let $s_{m}=\sum_{k=1}^{m} c_{k}$ be the sequence of partial sums of (37). We define $\varepsilon$-sequence by

$$
\begin{aligned}
\varepsilon_{0, m} & =0, \\
\varepsilon_{1, m} & =s_{m}, \\
& m=1,2,3, \ldots, \\
\varepsilon_{n+1, m} & =\varepsilon_{n-1, m+1}+\frac{1}{\left(\varepsilon_{n, m+1}-\varepsilon_{n, m}\right)},
\end{aligned}
$$


TABLE 1: Material properties.

\begin{tabular}{lccc}
\hline$k=386 \mathrm{~W} /(\mathrm{m} \mathrm{K})$ & $\alpha_{t}=1.78(10)^{-5} \mathrm{~K}^{-1}$ & $c_{E}=381 \mathrm{~J} /(\mathrm{kg} \mathrm{K})$ & $\eta=8886.73$ \\
$\mu=3.86(10)^{10} \mathrm{~kg} /\left(\mathrm{m} \mathrm{s}^{2}\right)$ & $\lambda=7.76(10)^{10} \mathrm{~kg} /\left(\mathrm{m} \mathrm{s}^{2}\right)$ & $\rho=8954 \mathrm{~kg} / \mathrm{m}^{3}$ & $T_{0}=293 \mathrm{~K}$ \\
$\varepsilon=0.0168$ & $\tau_{0}=0.025 \mathrm{~s}$ & $\tau_{1}=0.025 \mathrm{~s}$ & $\tau=0.025 \mathrm{~s}$ \\
\hline
\end{tabular}

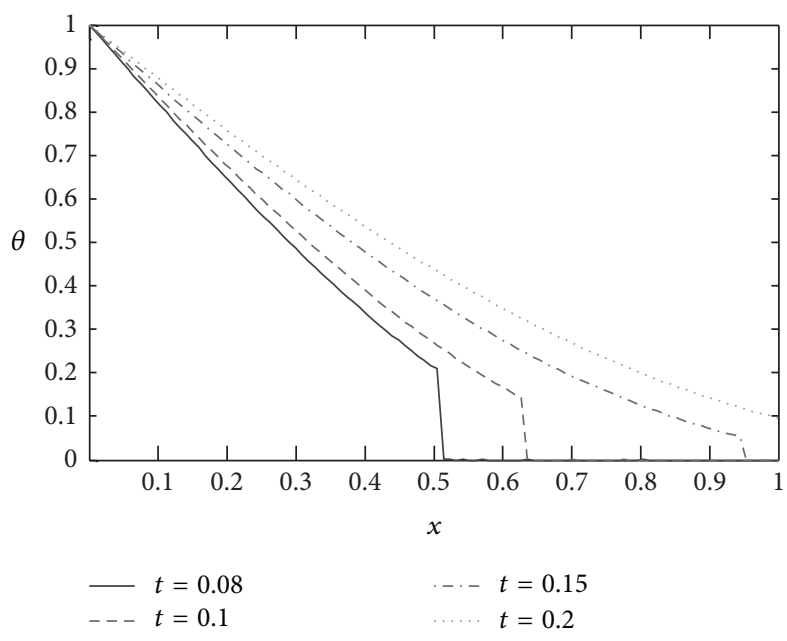

FIGURE 1: Temperature distribution for different time for $\alpha=\beta=1$.

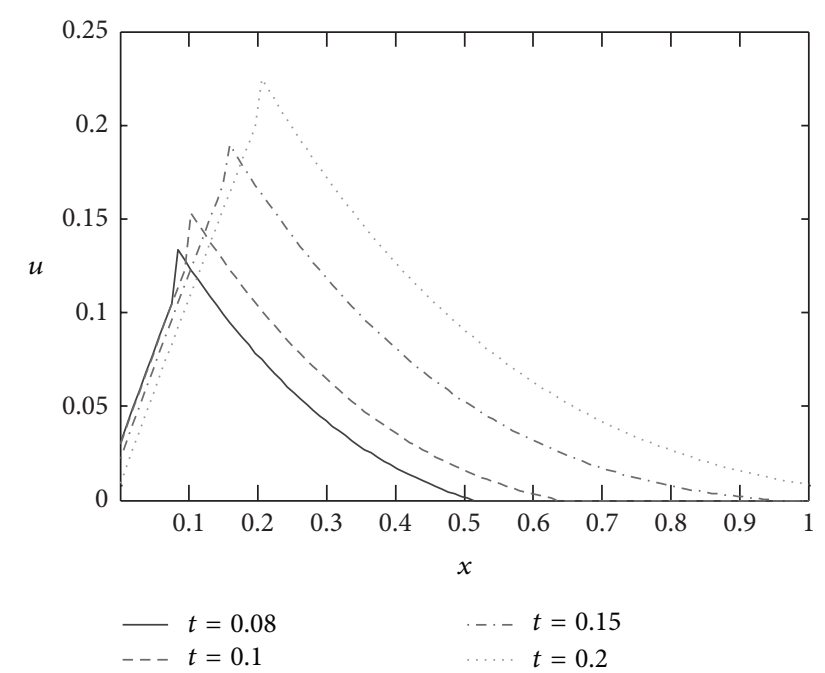

Figure 2: Displacement distribution for different times for $\alpha=\beta=$ 1.

It can be shown that [19] the sequence $\varepsilon_{1,1}, \varepsilon_{3,1}, \ldots, \varepsilon_{N, 1, \ldots}$ converges to $f(t)-c_{0} / 2$ faster than the sequence of partial sums.

\section{Numerical Results and Discussion}

In order to obtain the solutions for the field functions in the physical domain, we have applied the Laplace inversion formula mentioned in the above section. FORTRAN programming language was used on a personal computer. The

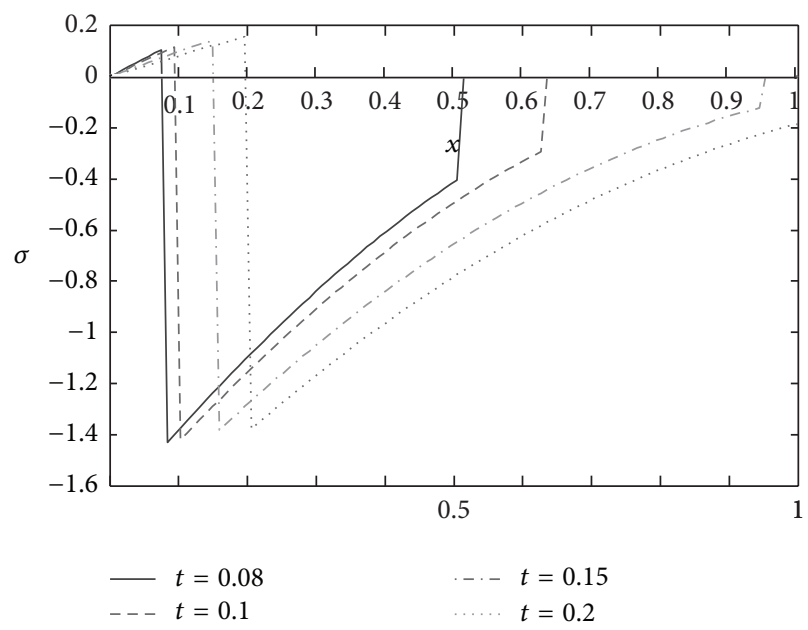

FIGURE 3: Stress distribution for different time when $\alpha=\beta=1$.

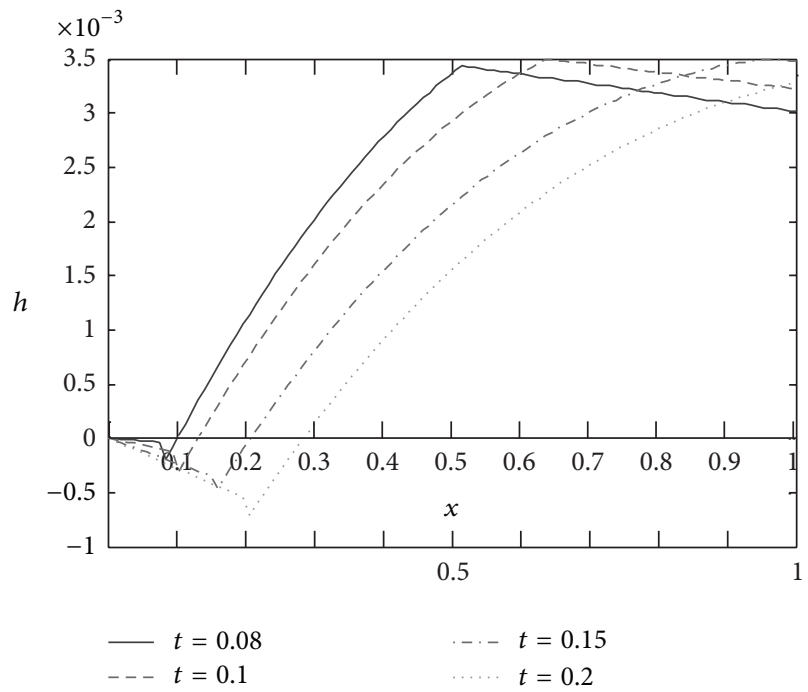

FIGURE 4: Induced magnetic field distribution for different time for $\alpha=\beta=1$.

accuracy maintained was 7 digits for the numerical program. For computational purposes, a copper-like material has been taken into consideration. The values of the material constants are taken as in Table 1.

In order to investigate the effect of time on all field variables the computations have been carried out for $t=0.05$, $t=0.1, t=0.15$, and $t=0.2$. The results are displayed in Figures 1-5, for the temperature, displacement, stress, and magnetic and electric field distributions, respectively. 
TABLE 2: Wave fronts and stress jump sizes.

\begin{tabular}{lcccc}
\hline \multirow{2}{*}{ Time } & \multicolumn{2}{c}{ First wave front of the stress } & \multicolumn{2}{c}{ Second wave front of the stress } \\
& Location & Discontinuity gap size & Location & Discontinuity gap size \\
\hline 0.08 & $x=0.0748$ & 1.5341611 & $x=0.5046729$ & 0.4026305 \\
0.1 & $x=0.0935$ & 1.5374964 & $x=0.6261683$ & 0.2926325 \\
0.15 & $x=0.1495327$ & 1.5193814 & $x=0.9439253$ & 0.1216954 \\
0.2 & $x=0.1962617$ & 1.5351866 & $x=0.7476634$ (reflected) & 0.0026 \\
\hline
\end{tabular}

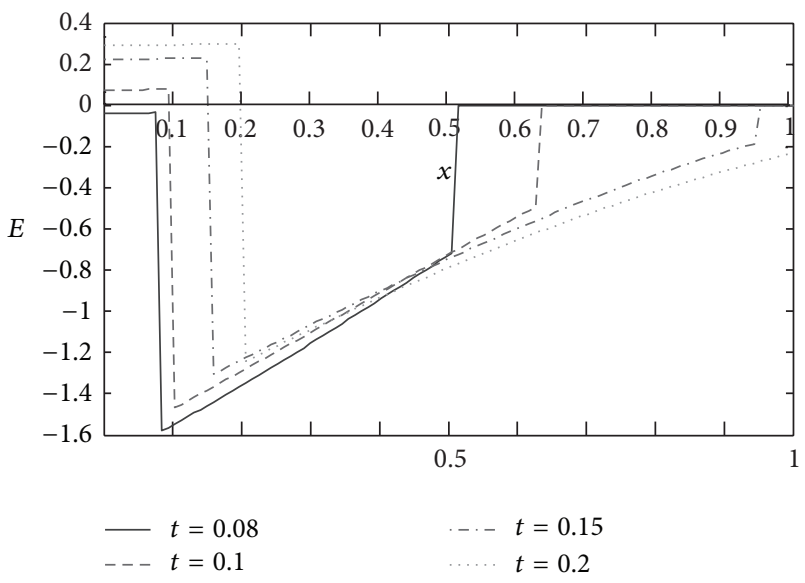

FIGURE 5: Induced electric field distribution for different time for $\alpha=\beta=1$.

Next, to study the effect of the fractional parameter $\alpha$, we take $\alpha=0.01, \alpha=0.2, \alpha=0.5$, and $\alpha=1$ when $t=0.1$ and $\beta=1$. This gives us Figures $6-10$, for the temperature, displacement, stress, magnetic field, and electric field distributions, respectively.

The remaining figures illustrate the effect of the fractional parameter $\beta$ on the field variables. Figures 11-14 describe the displacement, stress component, magnetic field, and electric field distributions for different values of $\beta$; namely, $\beta=0.2$, $\beta=0.5, \beta=0.9$, and $\beta=1$ when $t=0.1$ and $\alpha=1$. Here we notice that the fractional parameter $\beta$ has no effect on the temperature distribution.

The conclusions from Figures 1-14 can be summarized as follows:

(1) All of the physical variables have a finite speed of wave propagation for all times when $\alpha=\beta=1$. The speed of propagation for other values of $\alpha$ and $\beta$ needs further theoretical investigation.

(2) As is apparent from the order of the differential equation, we have two waves. The locations of the two wave fronts are the same for all functions considered. These wave fronts appear as a jump (discontinuity) in the case of the temperature, stress, and the intensity of the electric field. On the other hand, the other two functions, namely, the displacement and the intensity of the magnetic fields, are continuous. The wave fronts, in this case, appear as a cusp signifying a discontinuous first derivative. Note that the first

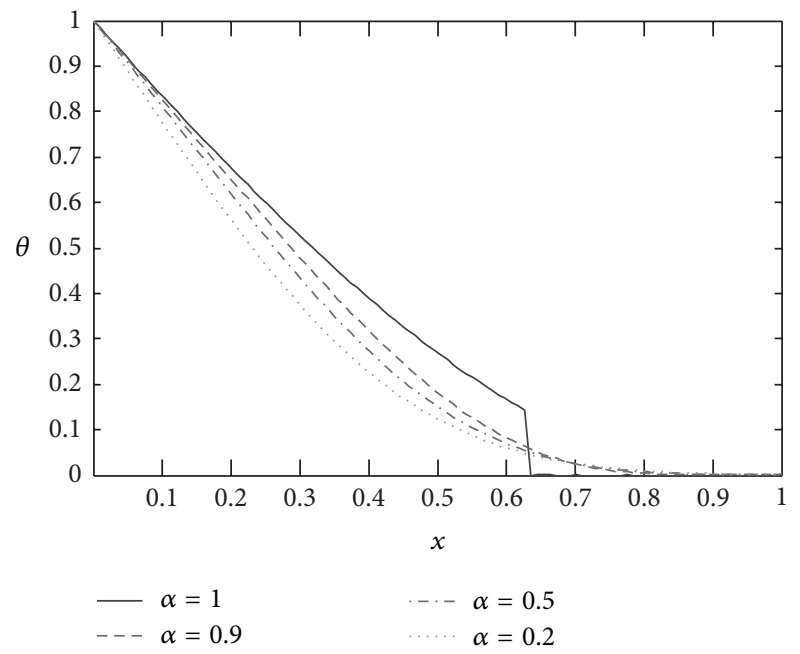

Figure 6: Temperature distribution for different $\alpha$ for $\beta=1$ at $t=$ 0.1 .

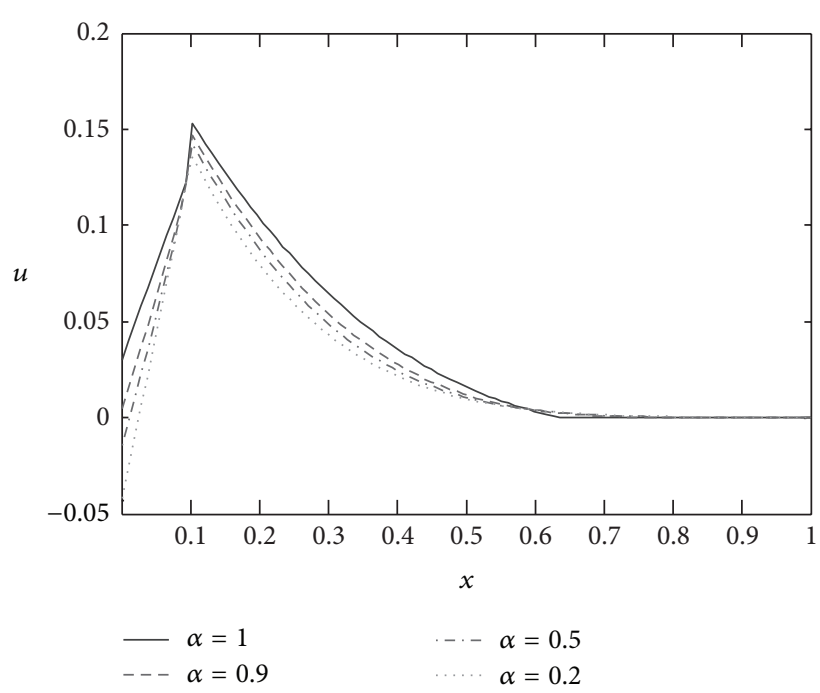

FIGURE 7: Displacement distribution for different $\alpha$ for $\beta=1$ at $t=$ 0.1 .

jump in the temperature is too small to appear in the graph. For $\alpha=\beta=1$, the waves propagate into the medium from the position $x=0$ to fill a finite part of the region that expands with the passage of time. We note that, for $t=0.2$, this region 


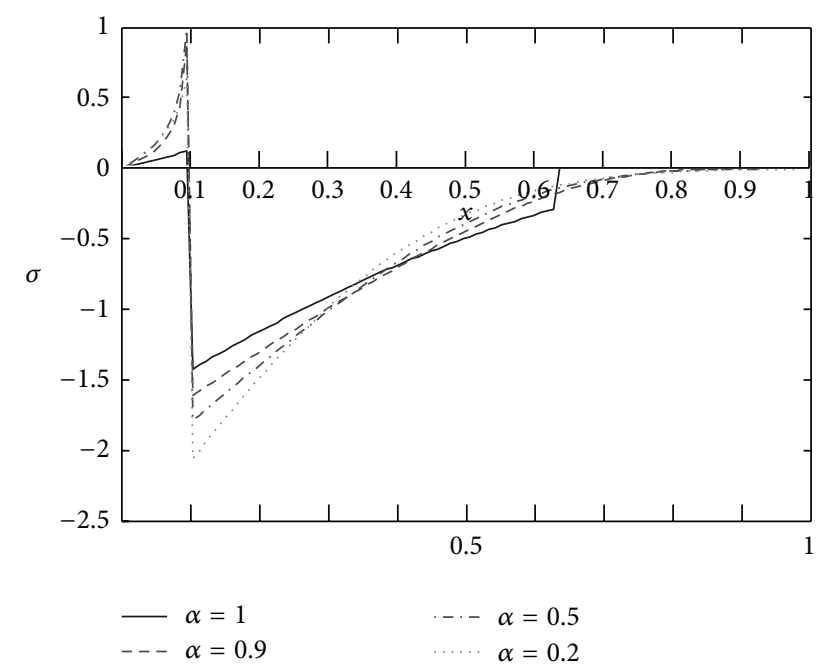

FIgURE 8: Stress distribution for different $\alpha$ for $\beta=1$ at $t=0.1$.

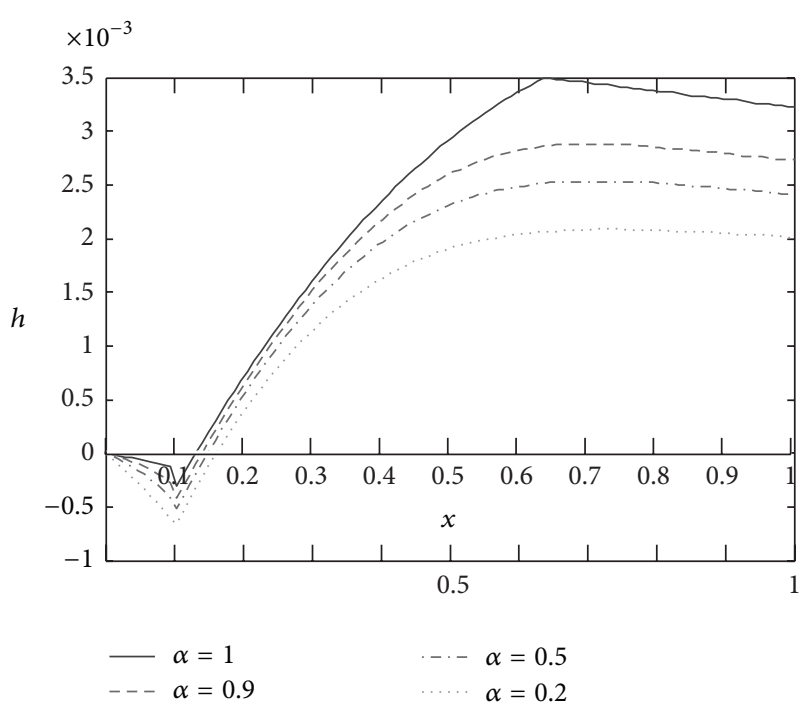

FIGURE 9: Induced magnetic field distribution for different $\alpha$ for $\beta=$ 1 at $t=0.1$.

has filled the entire body of the plate. The second wave has been reflected from the other side of the plate. The positions of the wave fronts for the stress and electric field distributions as well as the size of their jump discontinuities can be found in Tables 2 and 3.

(3) From Figure 3, we notice that for the displacement, the first wave front is located at the peaks of this function. The location of these peaks for different times is introduced in Table 4.

Also, we note here that the magnitude of peaks increases with time.

(4) It is observed from Figures 6-10 that the field variables $\theta, u, \sigma, h$, and $E$ are strongly affected by the fractional parameter $\alpha$. Increasing the fractional parameter $\alpha$ produces an increase in the peaks of the displacement and decreases the stress gap size at the location of the first wave front. Similar

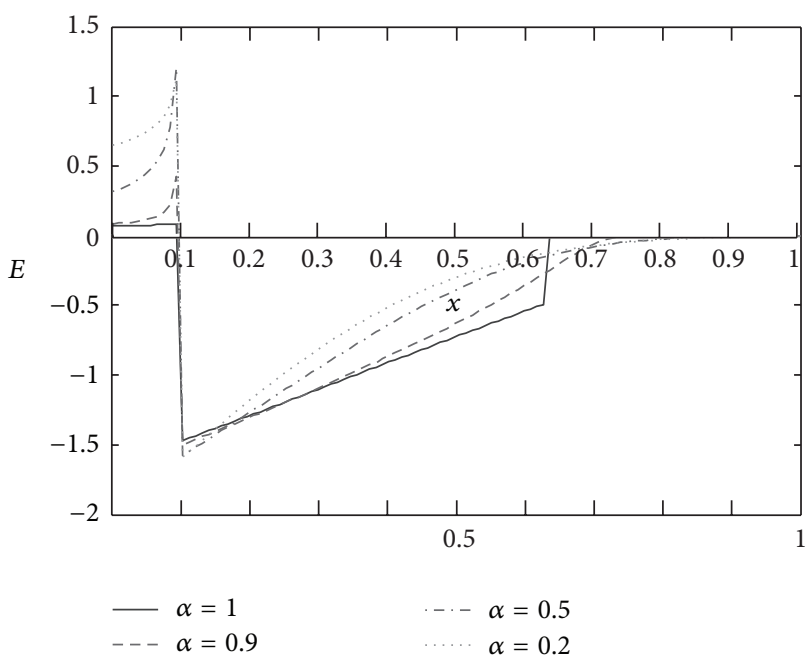

FIgURE 10: Induced electric field distribution for different $\alpha$ for $\beta=1$ at $t=0.1$.

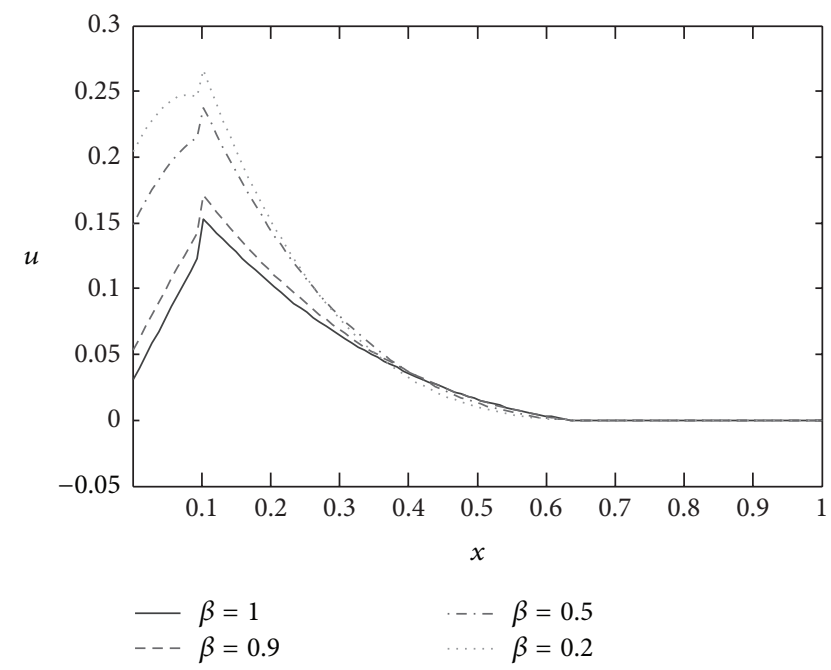

FIgURE 11: Displacement distribution for different $\beta$ for $\alpha=1$ at $t=0.1$.

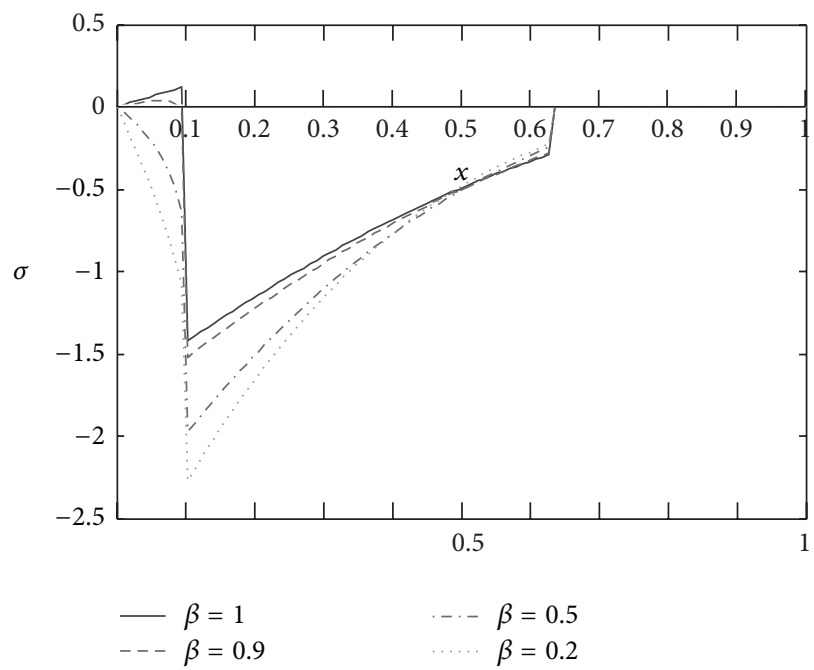

Figure 12: Stress distribution for different $\beta$ for $\alpha=1$ at $t=0.1$. 
TABLE 3: Wave fronts and electric field jump sizes.

\begin{tabular}{lcccc}
\hline \multirow{2}{*}{ Time } & \multicolumn{2}{c}{ First wave front of the electric field } & \multicolumn{2}{c}{ Second wave front of the electric field } \\
& Location & Discontinuity gap size & Location & Discontinuity gap size \\
\hline 0.08 & $x=0.0748$ & 1.5456 & $x=0.5046729$ & 0.7192609 \\
0.1 & $x=0.0935$ & 1.5477 & $x=0.6261683$ & 0.4947222 \\
0.15 & $x=0.1495327$ & 1.5299 & $x=0.7476634$ (reflected) & 0.1858727 \\
0.2 & $x=0.1962617$ & 1.541777 & & 0.0069 \\
\hline
\end{tabular}

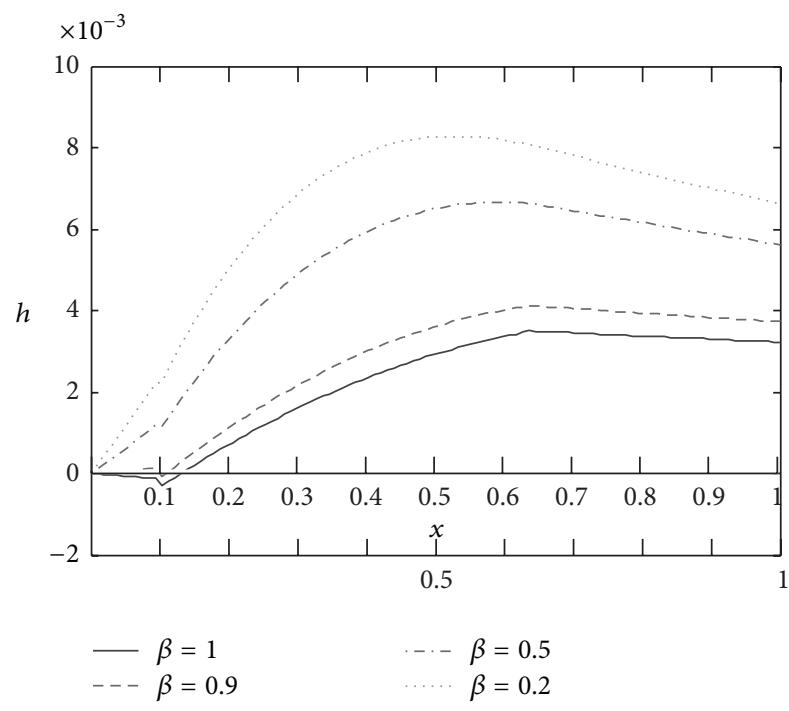

FIGURE 13: Induced magnetic field distribution for different $\beta$ for $\alpha=1$ at $t=0.1$.

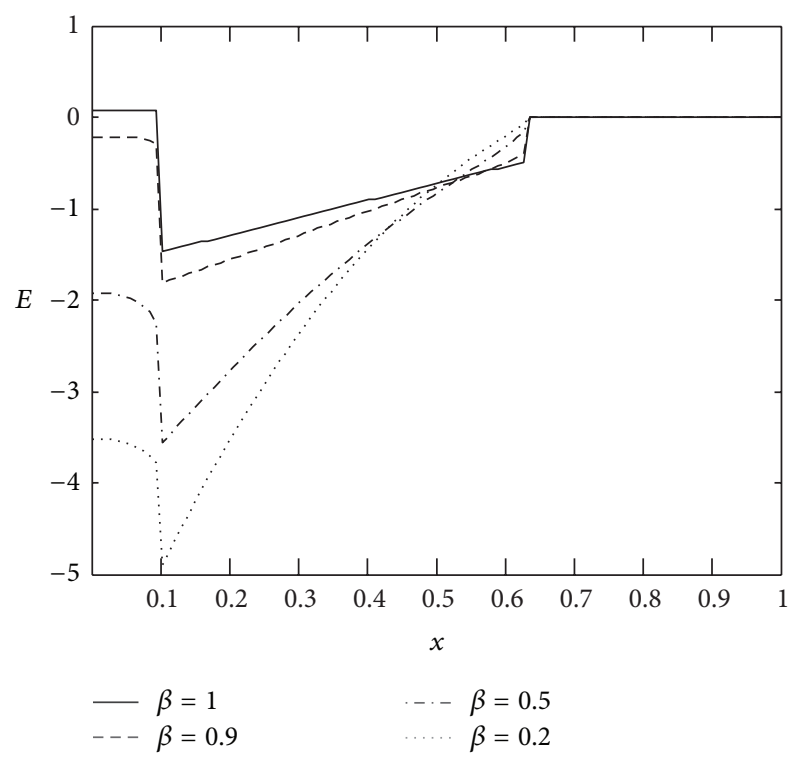

Figure 14: Induced electric field distribution for different $\beta$ for $\alpha=1$ at $t=0.1$.

effects of the parameter $\beta$ on $u, \sigma, h$, and $E$ are also clear from Figures 11-14.
TABLE 4: Wave fronts and displacement peak sizes.

\begin{tabular}{|c|c|c|}
\hline \multirow[b]{2}{*}{ Time } & \multicolumn{2}{|c|}{ First wave front location (peak) } \\
\hline & $\begin{array}{c}\text { Location of 1st } \\
\text { wave front }\end{array}$ & $\begin{array}{c}\text { Maximum value } \\
\text { at peak }\end{array}$ \\
\hline 0.08 & 0.0748 & 0.133331 \\
\hline 0.1 & 0.093457945 & 0.1530982 \\
\hline 0.15 & 0.1495327 & 0.1892859 \\
\hline 0.2 & 0.1962617 & 0.224686 \\
\hline
\end{tabular}

\section{Competing Interests}

The author declares that there are no competing interests.

\section{References}

[1] I. Podlubny, Fractional Differential Equations, Academic Press, New York, NY, USA, 1999.

[2] J. Klafter, S. C. Lim, and R. Metzler, Fractional Dynamics: Recent Advances, World Scientific, Singapore, 2011.

[3] R. Hilfer, Applications of Fractional Calculus in Physics, World Scientific Publishing, 2000.

[4] V. E. Tarasov, Fractional Dynamics: Applications of Fractional Calculus to Dynamics of Particles, Fields and Media, Higher Education Press, 2011.

[5] Y. Z. Povstenko, "Fractional heat conduction equation and associated thermal stress," Journal of Thermal Stresses, vol. 28, no. 1, pp. 83-102, 2004.

[6] Y. Z. Povstenko, "Fractional radial heat conduction in an infinite medium with a cylindrical cavity and associated thermal stresses," Mechanics Research Communications, vol. 37, no. 4, pp. 436-440, 2010.

[7] H. H. Sherief, A. M. A. El-Sayed, and A. M. Abd El-Latief, "Fractional order theory of thermoelasticity," International Journal of Solids and Structures, vol. 47, no. 2, pp. 269-275, 2010.

[8] W. E. Raslan, "Application of fractional order theory of thermoelasticity to a $1 \mathrm{D}$ problem for a spherical shell," Journal of Theoretical and Applied Mechanics, vol. 54, no. 1, pp. 295-304, 2016.

[9] W. E. Raslan, "Application of fractional order theory of thermoelasticity to a $1 \mathrm{D}$ problem for a cylindrical cavity," Archives of Mechanics, vol. 66, no. 4, pp. 257-267, 2014.

[10] W. E. Raslan, "Application of fractional order theory of thermoelasticity in a thick plate under axisymmetric temperature distribution," Journal of Thermal Stresses, vol. 38, no. 7, pp. 733743, 2015.

[11] H. Sherief and A. M. Abd El-Latief, "Effect of variable thermal conductivity on a half-space under the fractional order 
theory of thermoelasticity," International Journal of Mechanical Sciences, vol. 74, pp. 185-189, 2013.

[12] Y. Wang, D. Liu, Q. Wang, and J. Zhou, "Effect of fractional order parameter on thermoelastic behaviors of elastic medium with variable properties," Acta Mechanica Solida Sinica, vol. 28, no. 6, pp. 682-692, 2015.

[13] M. A. Ezzat, "Magneto-thermoelasticity with thermoelectric properties and fractional derivative heat transfer," Physica B: Condensed Matter, vol. 406, no. 1, pp. 30-35, 2011.

[14] G. Jumarie, "Derivation and solutions of some fractional BlackScholes equations in coarse-grained space and time. Application to Merton's optimal portfolio," Computers \& Mathematics with Applications, vol. 59, no. 3, pp. 1142-1164, 2010.

[15] F. Hamza, A. M. Abd El-Latief, and M. Fayik, "Memory time effect on electromagnetic-thermoelastic materials," Journal of Electromagnetic Waves and Applications, vol. 29, no. 4, pp. 474501, 2015.

[16] F. Hamza, M. Abdou, and A. M. Abd El-Latief, "Generalized fractional thermoelasticity associated with two relaxation times," Journal of Thermal Stresses, vol. 37, no. 9, pp. 1080-1098, 2014.

[17] F. Hamza, A. M. Abd El-Latief, and W. Khatan, "Thermomechanical fractional model of two immiscible TEMHD," Advances in Materials Science and Engineering, vol. 2015, Article ID 391454, 16 pages, 2015.

[18] F. Hamza, A. M. Abd El-Latief, and M. Abdou, "1D applications on fractional generalized thermoelasticity associated with two relaxation times," Mechanics of Advanced Materials and Structures, vol. 23, no. 6, pp. 689-703, 2016.

[19] G. Honig and U. Hirdes, "A method for the numerical inversion of Laplace transforms," Journal of Computational and Applied Mathematics, vol. 10, no. 1, pp. 113-132, 1984. 

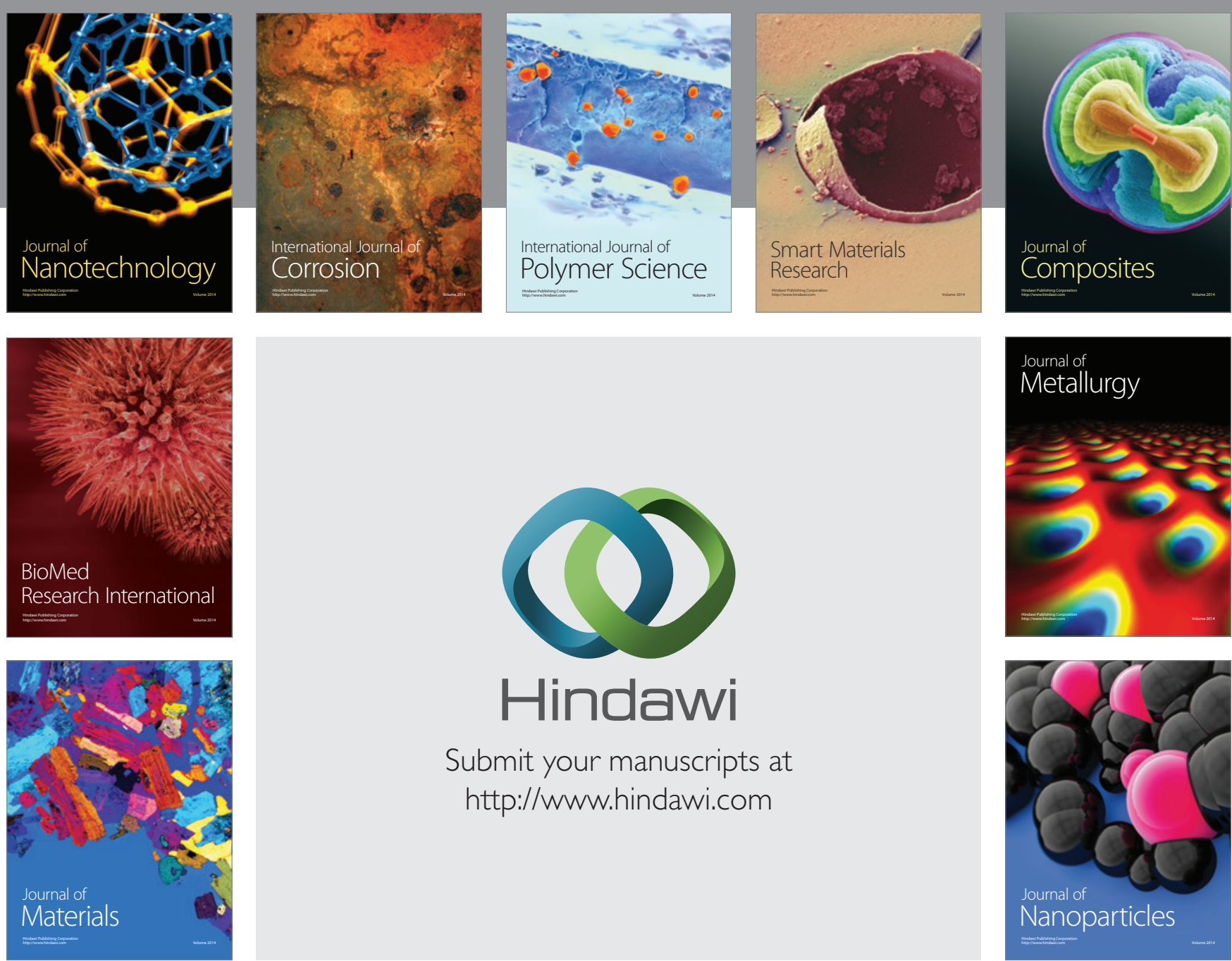

\section{Hindawi}

Submit your manuscripts at

http://www.hindawi.com

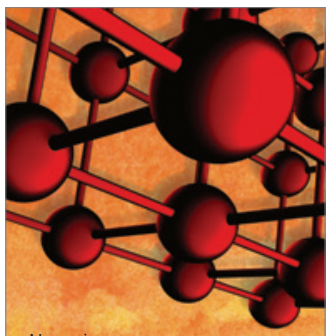

Materials Science and Engineering
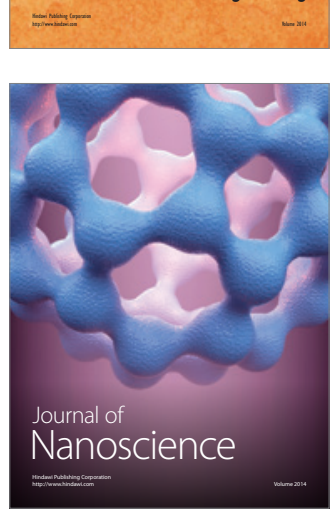
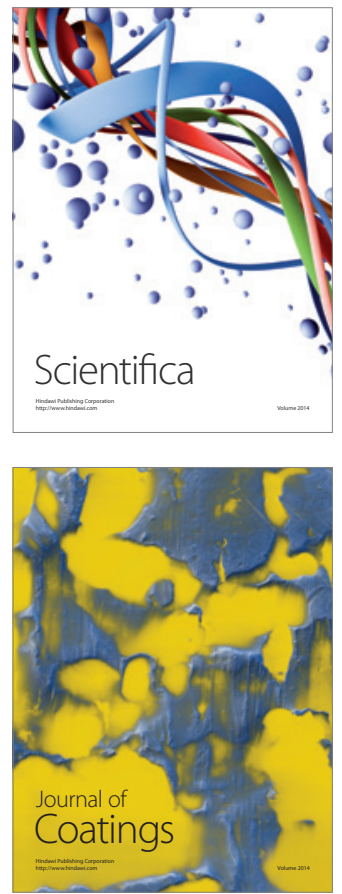
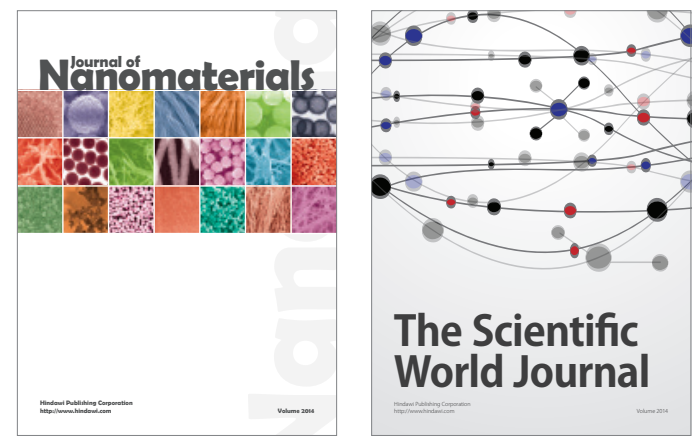

The Scientific World Journal
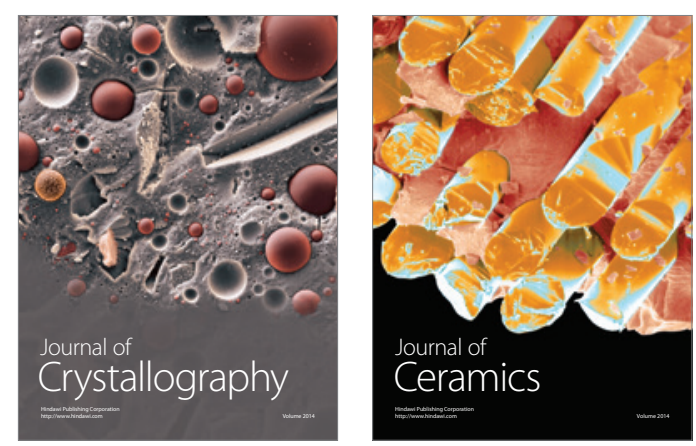
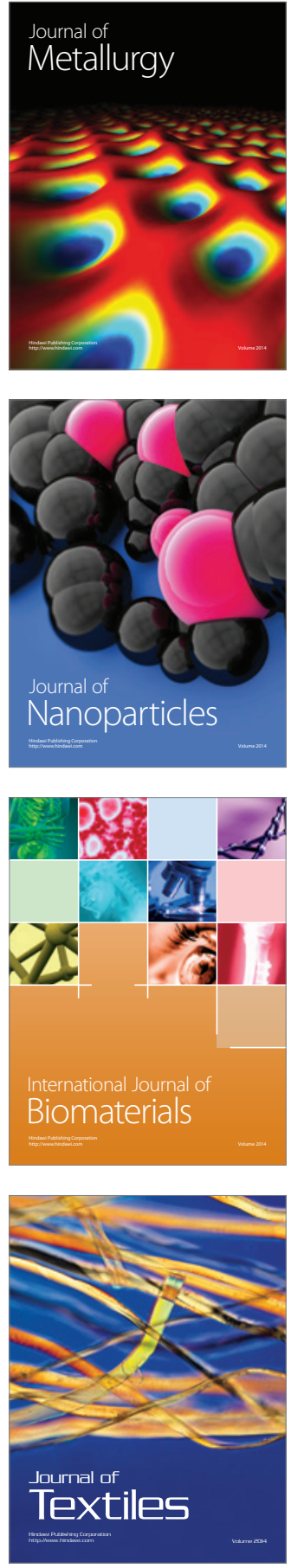